\title{
STABILITY OF A CONSTANT MEAN CURVATURE SURFACE IN $\mathrm{R}^{3}$
}

\author{
Sung Eun KoH
}

We solve the question raised by Barbosa and do Carmo as to whether there exists a complete, noncompact stably immersed surface in $R^{3}$ with nonzero constant mean curvature. We show that such a surface is necessarily minimal, that is, its mean curvature is zero.

\section{Introduction.}

Let $M$ be a 2-dimensional orientable, connected non-compact manifold and let $x: M \rightarrow \mathbb{R}^{3}$ be an immersion with constant mean curvature $h$. A domain $D \subset M$ with compact closure $\bar{D}$ is stable if

$$
\int\left[\left|v_{M} u\right|^{2}-\left(4 h^{2}-2 k\right) u^{2}\right] d M \geqq 0
$$

for all piecewise smooth functions $u$ with supports in $\vec{D}$ and satisfying the "volume" restriction $\int u d M=0$, where $k$ is the intrinsic Gaussian curvature of $M, d M$ is the area element of the induced metric, $\left|\nabla_{M} \mu\right|$ is the magnitude of the gradient of $u$ with respect to the induced metric. The immersion $x$ is stable if every such $D$ is stable.

It should be remarked that our definition of stability is different

Received 8 July 1986.

Copyright Clearance Centre, Inc. Serial-fee code: 0004-9729/87 $\$ A 2.00+0.00$. 
from that in the case of a minimal immersion, but in the case that the mean curvature $h$ is a nonzero constant, our definition is more useful in a sense as Barbosa and do Carmo demonstrated in [2]. In the same paper, they proved that if the compact $n$-dimensional manifold $M$ is immersed in $I^{n+1}$ with nonzero constant mean curvature and the immersion is stable, $M$ is a sphere and then conjectured that there is no complete stable immersion of a noncompact surface with nonzero constant mean curvature. The purpose of this paper is to show that their conjecture is true.

In fact we prove

THEOREM. Let $x: M \quad \boldsymbol{R}^{3}$ be an immersion of a non-compact 2dimensional manifold with constant mean curvature $h$ and $M$ is complete in the induced metric. If $x$ is stable, then $h=0$, that is, $x$ is a minimal immersion.

our theorem is related to the theorem of Chern [3] that if $M$ is a graph of a function defined over $\mathbb{R}^{n}$ with constant mean curvature, then the mean curvature is zero. It is also related to the recent result of Mori [6] that if the stability inequality (*) holds for all piecewise smooth functions with compact supports (without the "volume" restriction $\left.\int u d M=0\right), M$ is a plane.

2. Proof of the theorem.

For convenience, let ${ }_{D}$ denote the set of all piecewise smooth functions with supports in $\bar{D}$ and $F_{D}=\left\{u \in H_{D} \mid \int u d M=0\right\}$. We start from the following simple observation. If the stability inequality (*) holds not only for $u \in F_{D}$ but also for $u \in H_{D}$ for every bounded domain $D, M$ is a plane by the previously remarked result of Mori. So, let us assume that there is a bounded domain $K$ such that $(*)$ does not hold for some $v \in H_{K}$. For any bounded domain $D$ with $D \cap K=\phi$ and for $u \in H_{D}$, define $\tilde{u}=a v-b u, a=\int u d M, b=\int v d M$. Since $\tilde{u} \in F_{\tilde{D}}$ for some $\tilde{D} \supset D \cup K, \tilde{u}$ satisfies the stability inequality (*), 


$$
\begin{aligned}
0 \leqq & \int\left(\left|\nabla_{M} \tilde{u}\right|^{2}-\left(4 h^{2}-2 k\right) \tilde{u}^{2}\right) d M \\
= & a^{2} \int\left(\left|\nabla_{M} \nu\right|^{2}-\left(4 h^{2}-2 k\right) v^{2}\right) d M \\
& +b^{2} \int\left(\left|\nabla_{M} \mu\right|^{2}-\left(4 h^{2}-2 k\right) u^{2}\right) d M .
\end{aligned}
$$

since $\int\left(\left|\nabla_{M} v\right|^{2}-\left(4 h^{2}-2 k\right) v^{2}\right) d M<0$ by assumption, it follows that

$$
\int\left(\left|\nabla \mu^{\mu}\right|^{2}-\left(4 h^{2}-2 k\right) u^{2}\right) d M>0
$$

for all $u \in H_{D}$ with $D \cap K=\phi$. This fact implies that for every bounded domain $D$ with $D \cap K=\phi$, the first eigenvalue $\lambda_{1}(D)$ of the operator $\Delta_{M}+\left(4 h^{2}-2 k\right)$ is nonnegative, where $\Delta_{M}$ is the metric Laplacian acting on functions. We note that $\lambda_{1}(D)$ is characterized variationally as

$$
\lambda_{1}(D)=\inf \left\{\frac{\int\left(\left|\nabla_{M} u\right|^{2}-\left(4 h^{2}-2 k\right) u^{2}\right) d M}{\int u^{2} d M} \mid u \in H_{D}\right\}
$$

(A) In this section, we shall prove the theorem when the area of $M$ is finite.

Fix a point $p \in K$ and let $r$ be the distance from $P$ and $B_{R}$ be a geodesic ball with radius $R$ around $P$ and assume $K \subset B_{R_{0}}$ for some $R_{0}$. For a domain $D$, let $\mu_{1}(D)$ denote the first eigenvalue of the Dirichlet eigenvalue problem for Laplacian $\Delta_{M}$. Since the Lipschitz function $g(r)=(r-2 R)\left(x-R_{0}\right)$ is zero on the boundary of $B_{2 R} \sim B_{R_{0}}$, we have

$$
\begin{aligned}
\mu_{1}\left(B_{2 R} \sim B_{R_{0}}{ }^{\prime}\right. & \leqq \int_{B_{2 R} \sim B_{R_{0}}}\left|\nabla_{M} g\right|^{2} d M / \int_{B_{2 R} \sim B_{R_{0}}} g^{2} d M \\
& \leqq \int_{B_{2 R} \sim B_{R_{0}}}\left|\nabla_{M} g\right|^{2} d M / \int_{B_{R} \sim B_{R_{1}}} g^{2} d M
\end{aligned}
$$


for sufficiently large $R$ and some fixed $R_{1}>R_{0}$. Since the denominator is of order $R^{4}$ and the numerator is of order $R^{2}$ and since the area of $M$ is finite, letting $R$ to infinity, we can see that $\mu_{1}\left(B_{2 R} \sim B_{R_{0}}\right)$ converges to zero. If the mean curvature $h$ is not zero, $\mu_{1}\left(B_{R} \sim B_{R_{0}}{ }^{\prime}\right.$ $\leqq h^{2}$ for some large $R$. Since $2 h^{2}-k=2 \cdot\left(\frac{k_{1}+k_{2}}{2}\right)^{2}-k_{1} k_{2}=\frac{1}{2}\left(k_{1}^{2}+k_{2}^{2}\right) \geqq 0$ where $k_{1}, k_{2}$ are principal curvatures, we have from (1) that

$$
\lambda_{1}\left(B_{2 R} \sim B_{R_{0}}^{\prime} \leqq \mu_{1}\left(B_{2 R} \sim B_{R_{0}}\right)-2 h^{2} \leqq h^{2}-2 h^{2}=-h^{2}<0,\right.
$$

and since $\left(B_{2 R} \sim B_{R_{0}}\right) \cap K=\phi$, we get a contradiction.

(B) In this section, we shall consider the case when the area of $M$ is infinite.

With the natural complex structure given by the immersion, we can regard $M$ as a Riemann surface. Since $M$ is non-compact, the universal covering of $M$ is the complex plane $C$ or the unit disc $U$ in $C$ by the uniformization theorem.

(B.1) Let us first consider the case when $M$ is covered by $C$. Then $M$ is conformally equivalent to a cylinder or $M$ is conformally equivalent to the plane $C$ (see page 230 of [7]). In any case, let $z=x+i y$ denote a complex coordinate for $M$ so that $|d z|^{2}=d x^{2}+d y^{2}$ is the flat metric on $M$ and the metric induced by the immersion is $d s^{2}=\mu(z)|d z|^{2}$.

Fix a point $p \in K$ and let $r$ be the distance from $p$ taken with respect to the flat metric, $B_{R}=\{x \in M \mid r(x)<R\}$. Assume $K \subset B_{R_{0}}$ for some $R_{0} \cdot$ Fix $R_{1}>R_{0}$ and for any $R>R_{1}$, choose a smooth function $f(r)$ so that $f(r)=1$ for $R_{1} \leqq r \leqq R, f(r)=0$ for $r \leqq R_{0}, r \geqq 2 R$, $f^{\prime}(r) \geqq 0$ for all $r,\left|f^{\prime}(r)\right| \leqq 3 / R_{1}-R_{0}$ for $r \leqq R_{1}$ and $\left|f^{\prime}(r)\right| \leqq 3 / R$ for $r \geqq R$. Substituting this $f$ for $u$ in $(*)$ and by the conformal invariance of the Dirichlet integral we have 


$$
\begin{aligned}
& \left(3 / R_{1}-R_{0}\right)^{2} \int_{R_{0} \leq r \leq R_{1}} d x d y+(3 / R)^{2} \int_{\substack{R \leq r \leq 2 R \\
=}} d x d y \\
& \geqq \int_{B_{2 R} \sim B_{R_{0}}}\left(4 h^{2}-2 k\right) f^{2} d M \geqq \int_{B_{R} \sim B_{R_{1}}}\left(4 h^{2}-2 k\right) d M \\
& \geqq 2 h^{2} \int_{B_{R^{\sim B_{R}}}} d M=2 h^{2} \quad \text { (Area of }\left(B_{R} \sim B_{R_{1}}\right) \text { ). }
\end{aligned}
$$

Since the integral $\int_{\substack{R \leq r \leq 2 R \\=}} d x d y$ has growth by a constant times $R$ in the cylinder case and by a constant times $R^{2}$ in the plane case, letting $R$ infinity, we can see from the above inequality that the area of $M$ is finite, which is a contradiction.

(B.2) Now, let us consider the case then the unit disc $U$ covers $M$.

Since Smale's version of the Morse index theorem holds for the immersion $x: M \rightarrow R^{3}$ (see [2]), we can show that the immersion $x \circ \pi: U \rightarrow \mathbb{R}^{3}$ is also stable (see pages $521-526$ of [1]) where $\pi$ is a covering projection. So $\lambda_{I}(D) \geqq 0$ for all $D \subset U \sim K$ for some bounded domain $K \subset U$. Taking the same function $f$ as in the case of the universal covering $C$ in (B.1) where, $r$ is a distance with respect to the induced metric and duplicating Fischer-Colbrie and schoen (see section 2 of [5]) and noting that the area of $U$ in the complete metric given by the immersion $x \circ \pi$ is infinite, we can show that there is a domain ${ }^{B_{R}} \sim B_{R_{0}}$ for large $R$ such that $\lambda_{1}\left(B_{R} \sim B_{R_{0}}\right)<0$, which is a contradiction since $\left(B_{R} \sim B_{R_{0}}\right) \cap K=\phi$. Hence $U$ cannot cover $M$ and this completes the proof of the theorem.

Remark. There naturally arises the question, then, that what complete minimal surface in $\mathbb{R}^{3}$ is stable in our sense. We have shown that for some bounded domain $K \subset M, M \sim K$ is stable in the sense of minimal immersion, that is, the stability inequality ( $*$ ) holds for $u \in H_{D}$, not only for $u \in F_{D}$ with $D \cap K=\phi$. This case has been 
studied recently by Fischer-Colbrie. See [4] for details.

Note. After the preparation of this paper, M. do Carmo informed me that da Silveira had solved the same problem independently. His result is sharper. He showed that under the same condition $x(M)$ is a plane.

\section{References}

[1] J.L. Barbosa and M. do Carmo, "On the size of a stable minimal surfaces in $R^{3}$ ", Amer. Jour. Math. 98 (1976), 515-528.

[2] J.L. Barbosa and M. do Carmo, "Stability of hypersurfaces with constant mean curvature", Math. 2. 185 (1984), 339-353.

[3] S.s. Chern, "On the curvatures of a piece of hypersurface in euclidean space", Abh. Math. Sem. Univ. Hromburg 29 (1965), 77-91.

[4] D. Fischer-Colbrie, "On complete minimal surface with finite Morse index in three manifolds", Invent. Math. 82 (1985), 121-132.

[5] D. Fischer-Colbrie and R. Schoen, "The structure of complete stable minimal surface in 3-manifold of non-negative scalar curvature", Comm. Pure. App l. Math. 33 (1980), 199-211.

[6] H. Mori, "Stable constant mean curvature surfaces in $R^{3}$ and $H^{3}$ ", Trans.Amer. Math. Soc. 279 (1983), 671-687.

[7] G. Springer, Introduction to Riemann surfaces, (Reading, Mass. Addison-Wesley 1957).

Department of Mathematics,

Seoul. National University,

Seoul, 151, Korea. 